\title{
La medición del capital intelectual y su impacto en el rendimiento financiero en empresas del sector industrial en México
}

\author{
Mexico's industrial sector companies: A measurement of intellectual \\ capital and its impact on financial performance \\ Eleazar Villegas González*, Martín Aubert Hernández Calzada \\ y Blanca Cecilia Salazar Hernández
}

Universidad Autónoma del Estado de Hidalgo, México

Recibido el 3 de febrero de 2015; aceptado el 29 de junio de 2015

Disponible en Internet el 19 de noviembre de 2016

\section{Resumen}

En la actualidad el conocimiento es considerado como fuente primordial que propicia la creación y generación de ventaja competitiva y riqueza en las organizaciones. El factor humano como recurso intangible de una empresa se ha convertido en el principal generador de valor pese a que en la información financiera no se vea reflejado como tal. Tanto la gestión del conocimiento como el capital intelectual son 2 intangibles que se pueden medir en los reportes financieros de las empresas. En México, las empresas del sector industrial requieren impulsar la creación de valor añadido intelectual en función de sus propias capacidades, las cuales residen en su capital intelectual, y el coeficiente de valor añadido intelectual (VAIC ${ }^{\mathrm{TM}}$ ) es una herramienta que coadyuva al logro de este objetivo, que junto con sus componentes inciden e impactan positivamente en factores claves como lo son la rentabilidad financiera y la capitalización de mercado, y en el nivel del valor de las acciones.

(C) 2016 Universidad Nacional Autónoma de México, Facultad de Contaduría y Administración. Este es un artículo Open Access bajo la licencia CC BY-NC-ND (http://creativecommons.org/licenses/by-nc-nd/4.0/).

Códigos JEL: O34; L19; G39

Palabras clave: Capital intelectual; Coeficiente de valor añadido intelectual (VAIC ${ }^{\mathrm{TM}}$ ); Ventaja competitiva; Rendimiento financiero

\footnotetext{
* Autor para correspondencia.

Correo electrónico: eleazarvillegas@ hotmail.com (E. Villegas González).

La revisión por pares es responsabilidad de la Universidad Nacional Autónoma de México.
} 


\begin{abstract}
Currently knowledge is considered an essential resource to contribute to the creation and generation of competitive advantage in organizations. The human factor as an intangible resource of a company has become a main value generator, and even though both knowledge management and intellectual capital are intangibles that can be measured in the financial reports of companies, their position as main value generators is not reflected in financial information. In Mexico, industrial sector companies need to promote the creation of intellectual added value based on its own abilities, which reside on their intellectual capital. The value added intellectual coefficient $\left(\mathrm{VAIC}^{\mathrm{TM}}\right.$ ), with its components, is a tool that contributes to achieving this goal, and helps to identify the positive impact on key factors such as financial profitability, market capitalization and level of share value.

(C) 2016 Universidad Nacional Autónoma de México, Facultad de Contaduría y Administración. This is an open access article under the CC BY-NC-ND license (http://creativecommons.org/licenses/by-nc-nd/4.0/).
\end{abstract}

JEL classification: O34; L19; G39

Keywords: Intellectual capital; Value added intellectual coefficient (VAIC $\left.{ }^{\mathrm{TM}}\right)$; Competitive advantage; Financial performance

\title{
Introducción
}

En un periodo de aproximadamente 150 años el capitalismo y la tecnología han creado una civilización mundial sin precedentes gracias al significado que se le ha dado al conocimiento; si bien el capitalismo y las innovaciones técnicas no son algo nuevo, la velocidad en su difusión y su alcance mundial en las naciones sí lo han sido (Drucker, 1996). Las culturas occidentales y orientales han visto y vivido la transición del conocimiento del ser al hacer, de ser un bien privado a ser un bien público.

En la actualidad la persona es la portadora del conocimiento y por ende quien desempeña un papel fundamental en el escenario de los intangibles, por lo que el enfoque tradicional de verla como un recurso ha ido cambiando hacia una visión más sistémica, considerándola como protagonista de los procesos que tienen que ver con los intangibles (Díaz, de Liz y Rivero, 2009). Con la dinámica en la que se desenvuelve la sociedad del conocimiento o sociedad de la información, aquellos aspectos que tienen que ver con la información contable han cambiado radicalmente haciéndose exigible que dicha información refleje los elementos generadores de los beneficios de las organizaciones comprendidos en los activos intangibles, teniendo el problema de que los sistemas contables tradicionales solamente informan de aquellos elementos generadores de utilidades a través de los activos tangibles sobre hechos ya realizados que poco aportan para hechos del futuro, quedando de lado los intangibles como son el capital intelectual y el factor humano, los cuales no se publican en los estados financieros de una empresa, aunque sí se encuentran presentes en las organizaciones, y los cuales sí inciden en el desempeño financiero y no financiero de estas, siendo elementos determinantes claves de su posicionamiento en el mercado (Lev y Sougiannis, 1996; Palomo, 2003). El interés sobre este tema se ha ido acrecentando en la medida en que se desarrolla la nueva economía basada en tecnologías de la información y de servicios, en donde las empresas buscan crecer en base al conocimiento.

En el caso de México, las empresas del sector industrial requieren gestionar la creación de valor añadido intelectual y de riqueza en función de sus capacidades propias, las cuales residen en sus intangibles y en su capital intelectual, y que se han convertido en importantes recursos en 
la generación de ventajas competitivas, por lo que su medición resulta trascendental. Es por ello que en la presente investigación el coeficiente de valor añadido intelectual (VAIC ${ }^{\mathrm{TM}}$ ) junto con sus componentes eficiencia del capital empleado (CEE), eficiencia del capital humano (HCE) y eficiencia del capital estructural (SCE), se emplean como una herramienta que permite analizar su correlación y su influencia en factores como la rentabilidad, la capitalización de mercado y el valor de las acciones, en un grupo de empresas mexicanas del sector industrial que cotizan en la Bolsa Mexicana de Valores (Bolsa Mexicana de Valores, 2014), lo que permitirá identificarlos como elementos claves que contribuyen en su competitividad y éxito empresarial.

\section{Marco teórico}

\section{Teorías de la agencia y stakeholders}

La teoría de la agencia estudia la relación que se da entre los directivos (agentes) y los accionistas (principales), donde los agentes tienen la encomienda de maximizar el valor neto de la empresa lo cual representa el objetivo fundamental de los principales (Ross, 1973; Jensen y Meckling, 1976), considerados como los propietarios de ella, sin embargo, en la economía del conocimiento no tienen por qué existir propietarios bajo el enfoque tradicional. La empresa debe de referirse como una organización humana integrada por miembros en la que interactúan varios sujetos: inversionistas, directivos y trabajadores, y no únicamente como la suma de sus activos físicos y financieros (Fontrodona y Sison, 2007).

Llano (1997) señala que la empresa es el resultado de la combinación de 3 factores: el capital, la dirección y el trabajo, no debiendo por qué ser el capital el que más valor o peso tenga en una compañía. Drucker (2001) afirma que el activo más importante de una empresa es su capital humano siendo el conocimiento el principal elemento de la producción, que pertenece a los trabajadores y que además cuenta con la característica de ser transferible, por lo tanto, el trabajador y el accionista poseen un estatus similar, el primero al proporcionar capital humano y el segundo al abastecer de recursos financieros.

Por otro lado, la teoría de los stakeholders (Freeman, 1984; Vassolo, García y Weisz, 2007) define a la empresa como un grupo conformado por distintos participantes los cuales se encuentran alineados tras un objetivo en común, considerando entre dichos participantes a los inversionistas, directivos, empleados, proveedores, clientes, y a todas aquellas personas que pudieran tener un interés en las actividades propias de ella como son el gobierno, las organizaciones sindicales y la población local, destacando el hecho de que una empresa crea y distribuye valor de una manera sostenible a través de la coordinación de los diferentes grupos de stakeholders.

Desde estas 2 perspectivas, los directivos y accionistas de una empresa deben de diseñar e implementar sus procesos y estrategias encaminados a satisfacer los intereses de los diferentes grupos de stakeholders (Kochan y Rubinstein, 2000; Freeman, Wicks y Parmar, 2004) y no únicamente los suyos propios, buscando de esta manera el bien común de los diversos grupos que integran el entorno de una empresa.

\section{Gestión del conocimiento}

Para poder competir en los mercados globales la gestión del conocimiento resulta crucial para cualquier tipo de empresa, la producción de conocimiento solo se lleva a cabo a través de configuraciones de redes internas y externas que estas desarrollan. 
Tabla 1

Definiciones básicas de gestión del conocimiento

\begin{tabular}{ll}
\hline Autores & Definición \\
\hline Saint-Onge (1998) & $\begin{array}{l}\text { Es la habilidad de desarrollar, mantener, influenciar y renovar los activos } \\
\text { intangibles llamados capital de conocimiento o capital intelectual }\end{array}$ \\
Steward (1998) & $\begin{array}{l}\text { Es el conjunto de procesos que hacen que el capital intelectual de la } \\
\text { empresa crezca } \\
\text { Es el proceso sistemático de buscar, organizar, filtrar y presentar la } \\
\text { información con el objetivo de mejorar la comprensión de las personas } \\
\text { en un área específica de interés } \\
\text { Es la gestión y movilización de los activos intangibles de la empresa } \\
\text { sobre los que debe sustentarse la capacidad de aprendizaje y mejora } \\
\text { continua de la organización }\end{array}$ \\
& $\begin{array}{l}\text { Es hacer lo que se necesita para obtener lo máximo de los recursos } \\
\text { de conocimiento }\end{array}$ \\
\hline Becerra-Fernández y Sabherwal (2005) &
\end{tabular}

Fuente: Arias et al. (2007; p. 68); Monagas-Docasal (2012, p. 244).

Diversos autores se han dado a la tarea de conceptualizar el término gestión del conocimiento, con distintas perspectivas y enfoques, por lo que en la tabla 1 se presentan estas definiciones.

Aunado a estas definiciones, Arias, Cruz, Pedraza, Ordoñez y Herrera (2007) han señalado que la gestión del conocimiento se basa en aspectos tácticos y operacionales, facilitando y gestionando aquellas actividades relacionadas con él como es su creación, captura, transformación y uso, por lo que su función se centra en planificar, poner en marcha, operar y controlar programas y actividades que tienen que ver con el conocimiento, en tanto que para Monagas-Docasal (2012) resulta ser la base en la generación del capital intelectual.

Por su parte, Drucker (1993) ha destacado la manera en que el conocimiento ha reemplazado al capital financiero como principal condicionante de desarrollo en las sociedades contemporáneas, siendo el único recurso que garantiza en el presente y en el futuro la sustentabilidad económica, por lo que su gestión tiene que ver con la manera en que una empresa genera, comunica y aprovecha a su capital intelectual.

Bossi, Fuentes y Serrano (2005) reconocen que los recursos humanos de una entidad y el conocimiento son activos intangibles input fundamentales en la era del conocimiento. Por lo tanto, la gestión del conocimiento es un elemento clave y de vital importancia en las empresas, ya que obtiene y transforma la información en conocimiento útil, maximiza los recursos y contribuye a una mejora continua, generando con ello una ventaja competitiva.

\section{Capital intelectual}

Villarreal (2003) señala que los elementos que caracterizan a la nueva economía global son la era del conocimiento y la mentefactura (trabajador del conocimiento) en donde el capital intelectual se ha convertido en el factor estratégico del nuevo paradigma de la competitividad al ser inteligentes en la organización, flexibles en la producción y ágiles en la comercialización IFA.

Bueno, Salmador y Merino (2008) y Monagas-Docasal (2012) han recopilado una serie de definiciones del capital intelectual las cuáles se presentan en la tabla 2 manifestando que todas ellas muestran cierto grado de heterogeneidad y el estado aún emergente del concepto. 
Tabla 2

Definiciones básicas de capital intelectual

\begin{tabular}{|c|c|}
\hline Autores & Definición \\
\hline List (1841) & $\begin{array}{l}\text { El resultado de la acumulación de los descubrimientos, invenciones, mejoras, } \\
\text { perfeccionamientos y esfuerzos de todas las generaciones que nos han precedido } \\
\text { (capital intelectual de la raza humana) }\end{array}$ \\
\hline Kendrick (1961) & $\begin{array}{l}\text { El resultado de las inversiones en el descubrimiento y difusión del conocimiento } \\
\text { productivo }\end{array}$ \\
\hline Brooking (1996) & La combinación de activos intangibles que permiten a la empresa funcionar \\
\hline Edvinsson y Malone (1997) & $\begin{array}{l}\text { La posesión de conocimientos, experiencia aplicada, tecnología organizativa, } \\
\text { relaciones con los clientes y destrezas profesionales que proporcionan una ventaja } \\
\text { competitiva en el mercado }\end{array}$ \\
\hline Sveiby (1997) & $\begin{array}{l}\text { La combinación de activos intangibles que generan crecimiento, renovación, } \\
\text { eficiencia y estabilidad en la organización }\end{array}$ \\
\hline Bueno (2002) & $\begin{array}{l}\text { Representa la perspectiva estratégica de la «cuenta y razón» de los intangibles } \\
\text { de la organización }\end{array}$ \\
\hline Machado y Monagas (2004) & $\begin{array}{l}\text { Es la parte del proceso cognitivo en que la preponderancia la tienen las ideas más } \\
\text { que las emociones, las ideas y la forma en que se manifiestan las definiciones } \\
\text { y conceptos en sus diferentes grados de estructuración: estrategias, proyectos } \\
\text { de calidad, enfoques de procesos }\end{array}$ \\
\hline Rivero (2009) & $\begin{array}{l}\text { Es la sinergia de todos los conocimientos que reúne una organización, toda la } \\
\text { experiencia acumulada en sus integrantes, sus capacidades, destrezas, su } \\
\text { motivación y compromiso, valores, etc., aplicados al trabajo. Todo lo que ha } \\
\text { conseguido en términos de relaciones, procesos, descubrimientos, innovaciones, } \\
\text { presencia en el mercado e influencia y reconocimiento en la sociedad }\end{array}$ \\
\hline
\end{tabular}

Fuente: Bueno et al. (2008, p. 52); Monagas-Docasal (2012, p. 244).

De estas definiciones Bueno et al. (2008) han concluido que el concepto de capital intelectual:

- Indica el valor de la riqueza acumulada derivada del conocimiento o de un conjunto de activos de naturaleza intangible.

- Combina activos de naturaleza intangible, los cuales crean nuevo conocimiento. Este se transforma en competencias empresariales o en la creación de ventaja competitiva.

- Genera valor a la empresa y representa la nueva riqueza de las organizaciones y de las naciones.

- No suele reflejarse en los estados financieros de una empresa.

Por su parte, Monagas-Docasal (2012) hace una valoración sobre las definiciones expresadas por los diferentes autores, resaltando sus principales aspectos:

- Resulta una combinación de activos inmateriales que permiten funcionar a la empresa.

- Es la habilidad para transformar nuevas ideas en nuevos productos y servicios.

- Es la posesión de conocimientos, experiencias aplicadas, tecnología organizacional, relaciones con clientes y destrezas profesionales.

De todas estas definiciones y conceptos se puede concluir que el capital intelectual es un activo intangible no transferible que tiene la virtud de generar riqueza en una organización gracias a la combinación de elementos de naturaleza intangible así como de sus recursos humanos y estructurales, permitiendo capitalizar experiencias, transformando el conocimiento en una ventaja competitiva. 


\section{Métodos para medir el capital intelectual}

Por el valor que se le ha dado al capital intelectual dentro de las organizaciones se han construido una serie de modelos para su medición, dado que las medidas financieras resultan ser insuficientes para dirigir el proceso en la toma de decisiones en las empresas, y que los sistemas tradicionales de la contabilidad solo señalan los resultados de hechos ya ejecutados y que poco aportan para hechos del futuro; por tal motivo se hace necesario que estas estén acompañadas de medidas no financieras para determinar aquellos elementos que contribuyan a que las empresas sean más competitivas.

En la época de los años 80 un pequeño grupo de empresas entre las que se encuentran Skandia, Dow Chemicals y el Canadian Imperial Bank empezaron a vincular el término de capital intelectual al de activos intangibles, despertando su interés en aquellas empresas cuyos beneficios derivaban principalmente de la innovación y de los servicios intensivos del conocimiento (Sánchez, Melián y Hormiga, 2007).

La empresa sueca Skandia se dio cuenta de que las teorías tradicionales de la administración no reconocían que la fortaleza competitiva en una organización no radicaba en los activos contables, sino en otros factores derivados de los recursos humanos, por lo que en el año 1991 creó su propio departamento de capital intelectual, con el propósito de evaluarlo y dar cuenta de su verdadero valor y cómo este hacía que la empresa fuera más competitiva (Ramírez, 2007).

Uno de los precursores en la medición de los activos intangibles es Karl-Erik Sveiby (2010) quien ha asociado a los diferentes modelos que sirven para tal fin 4 métodos o enfoques, señalando que ninguno de ellos cumple con todos los propósitos, por lo que al momento de seleccionar alguno de ellos se debe de tomar en cuenta los aspectos relacionados con una situación en específico, el fin o hacia a quien van dirigidos.

En lo que se refiere al método de retorno sobre activos ROA, Sveiby (2010) señala que este es de gran utilidad al llevar a cabo comparativos entre empresas del mismo sector al basarse en normas contables ya establecidas, lo que permite ilustrar de una mejor manera el valor financiero de los activos intangibles; de acuerdo a esta clasificación se consideran 3 modelos, los cuales se muestran en la tabla 3.

De estos 3 modelos, el VAIC ${ }^{\mathrm{TM}}$ se ha seleccionado para medir el capital intelectual de un grupo de empresas mexicanas del sector industrial que cotizan en la Bolsa Mexicana de Valores por el periodo comprendido de 2006 a 2012.

\section{El modelo del coeficiente de valor añadido intelectual}

Pulic (2000) ha expresado que los modelos tradicionales de medición del desempeño en las organizaciones no han sido lo suficientemente adecuados en un contexto de la economía del conocimiento señalando que los indicadores básicos de la economía industrial en realidad no manifiestan cuánto y si se ha creado valor, afirmando que la creación de valor es el núcleo principal de los negocios modernos y que los sistemas contables son incapaces de proveer información relacionada con este valor añadido por sí mismos; por todo esto, a través del método VAIC ${ }^{\mathrm{TM}}$ sí se puede medir y supervisar la eficiencia en la creación de valor en una empresa utilizando las cifras reportadas en la contabilidad, asociadas a cada componente del capital intelectual y el capital empleado (capital físico y financiero), confirmando que la mayor eficiencia en la creación de valor se basa en el capital humano, como factor de creación de valor decisivo de los negocios modernos. 
Tabla 3

Método de retorno sobre activos

\begin{tabular}{|c|c|c|c|}
\hline Año & Modelo & Autor principal & Descripción \\
\hline 1995 & $\begin{array}{l}\text { Economic Value Added } \\
\left(\mathrm{EVA}^{\mathrm{TM}}\right)\end{array}$ & $\begin{array}{l}\text { Stern, Stewart y Chew } \\
\text { (1995) }\end{array}$ & $\begin{array}{l}\text { Calculado mediante el ajuste de las } \\
\text { utilidades de la firma publicadas con cargos } \\
\text { relacionados con los intangibles. Los } \\
\text { cambios en EVA }{ }^{\mathrm{TM}} \text { proporcionan un } \\
\text { indicador de si el capital intelectual de una } \\
\text { empresa es productivo o no. EVA }{ }^{\mathrm{TM}} \text { es } \\
\text { propiedad de la empresa de consultoría Stern } \\
\text { and Stewart y uno de los métodos más } \\
\text { comunes }\end{array}$ \\
\hline 1998 & $\begin{array}{l}\text { Value Added } \\
\text { Intellectual Coefficient } \\
\left(\text { VAIC }^{\mathrm{TM}}\right)\end{array}$ & Pulic (1998) & $\begin{array}{l}\text { Una ecuación que mide la cantidad y la } \\
\text { eficiencia del capital intelectual y el capital } \\
\text { empleado que crea valor basado en la } \\
\text { relación de } 3 \text { componentes principales: (1) el } \\
\text { capital empleado, (2) el capital humano y (3) } \\
\text { el capital estructural. VAIC TM } \\
\text { i = CEEI + HCEI + SCEI. }\end{array}$ \\
\hline 1999 & $\begin{array}{l}\text { Knowledge Capital } \\
\text { Earnings }\end{array}$ & Lev (1999) & $\begin{array}{l}\text { Las utilidades del capital del conocimiento } \\
\text { se calculan como la porción de las ganancias } \\
\text { normalizadas ( } 3 \text { años promedio de la } \\
\text { industria y el consenso de las estimaciones } \\
\text { de los analistas para el futuro) por encima de } \\
\text { las ganancias atribuibles a los activos en } \\
\text { libros. Las ganancias luego son utilizadas } \\
\text { para capitalizar el capital del conocimiento }\end{array}$ \\
\hline
\end{tabular}

Fuente: Elaboración propia siguiendo a Sveiby (2010).

Pulic (2004), Kujansivu y Lonnqvist (2007) y Laing, Dunn y Hughes-Lucas (2010) han estructurado metodológicamente 6 pasos para llegar al cálculo del VAIC ${ }^{\mathrm{TM}}$ los cuales quedan ilustrados en la tabla 4.

Abdulsalam, Al-Qaheri y Al-Khayyat (2011) han justificado la utilidad que tiene la aplicación del modelo VAIC ${ }^{\mathrm{TM}}$ enlistando las siguientes características:

- Se producen medidas cuantificables, objetivas y cuantitativas sin el requisito de alguna calificación subjetiva.

- Se emplean procedimientos relativamente simples y directos en el cálculo de coeficientes e índices, que pueden ser fácilmente entendibles, especialmente para aquellos hombres de negocios y empresarios acostumbrados a la información contable tradicional.

- Se utilizan medidas financieras para que cualquier tipo de indicadores o ratios calculados puedan ser empleados para la comparación con indicadores financieros tradicionales que comúnmente se encuentran en los negocios, los cuales se basan en unidades o medidas monetarias.

- Al capital humano -los trabajadores- se le considera como la fuente más importante de capital intelectual, lo cual concuerda con la mayoría de las definiciones que se encuentran en la literatura.

- Posee una importante trayectoria en su implementación y aplicación en investigaciones que tienen que ver con la valoración del capital intelectual en empresas que cotizan en bolsa en diferentes países por parte de investigadores. 
Tabla 4

Cálculo de VAIC ${ }^{\mathrm{TM}}$

\begin{tabular}{|c|c|c|c|}
\hline \multirow[t]{2}{*}{ Paso 1} & Cálculo del valor añadido (VA) & $\begin{array}{l}\text { Formula } \\
\mathrm{VA}=\mathrm{IT}-\mathrm{CV}\end{array}$ & $\begin{array}{l}\text { Variables } \\
\mathrm{IT}=\text { ingresos totales } \\
\mathrm{CV}=\text { costos de ventas }\end{array}$ \\
\hline & Fuente de consulta & \multicolumn{2}{|c|}{ Estado de pérdidas y ganancias y las notas a los estados financieros } \\
\hline \multirow[t]{2}{*}{ Paso 2} & $\begin{array}{l}\text { Cálculo del coeficiente de } \\
\text { eficiencia del capital humano } \\
\text { (HCE) }\end{array}$ & $\mathrm{HCE}=\mathrm{VA} / \mathrm{HC}$ & $\begin{array}{l}\mathrm{VA}=\text { valor añadido } \\
\mathrm{HC}=\text { sueldos y salarios (capital humano) }\end{array}$ \\
\hline & Fuente de consulta & \multicolumn{2}{|c|}{ Estado de pérdidas y ganancias y las notas a los estados financieros } \\
\hline \multirow[t]{2}{*}{ Paso 3} & $\begin{array}{l}\text { Cálculo del coeficiente de } \\
\text { eficiencia del capital estructural } \\
\text { (SCE) }\end{array}$ & $\mathrm{SCE}=\mathrm{SC} / \mathrm{VA}$ & $\begin{array}{l}\mathrm{SC}=\text { capital estructural } \\
\mathrm{SC}=\mathrm{VA}-\mathrm{HC} \\
\mathrm{VA}=\text { valor añadido }\end{array}$ \\
\hline & Fuente de consulta & \multicolumn{2}{|c|}{ Estado de pérdidas y ganancias y las notas a los estados financieros } \\
\hline \multirow[t]{2}{*}{ Paso 4} & $\begin{array}{l}\text { Cálculo del coeficiente de } \\
\text { eficiencia del capital intelectual } \\
\text { (ICE) }\end{array}$ & $\mathrm{ICE}=\mathrm{SCE}+\mathrm{HCE}$ & $\begin{array}{l}\text { SCE = coeficiente de eficiencia del capital } \\
\text { estructural } \\
\text { HCE = coeficiente de eficiencia del capital } \\
\text { humano }\end{array}$ \\
\hline & Fuente de consulta & \multicolumn{2}{|c|}{ Estado de pérdidas y ganancias y las notas a los estados financieros } \\
\hline \multirow[t]{2}{*}{ Paso 5} & $\begin{array}{l}\text { Cálculo del coeficiente de } \\
\text { eficiencia del capital empleado } \\
\text { (CEE) }\end{array}$ & $\mathrm{CEE}=\mathrm{VA} / \mathrm{CE}$ & $\begin{array}{l}\mathrm{VA}=\text { valor añadido } \\
\mathrm{CE}=\text { valor en libros de los activos netos } \\
\text { de una empresa }\end{array}$ \\
\hline & Fuente de consulta & \multicolumn{2}{|c|}{$\begin{array}{l}\text { Balance general, estado de pérdidas y ganancias y las notas a los } \\
\text { estados financieros }\end{array}$} \\
\hline \multirow[t]{2}{*}{ Paso 6} & $\begin{array}{l}\text { Cálculo del coeficiente de valor } \\
\text { añadido intelectual (VAIC }\end{array}$ & $\mathrm{VAIC}^{\mathrm{TM}}=\mathrm{ICE}+\mathrm{CEE}$ & $\begin{array}{l}\text { ICE = coeficiente de eficiencia del capital } \\
\text { intelectual } \\
\text { CEE = coeficiente de eficiencia del capital } \\
\text { empleado }\end{array}$ \\
\hline & Fuente de consulta & \multicolumn{2}{|c|}{$\begin{array}{l}\text { Balance general, estado de pérdidas y ganancias y las notas a los } \\
\text { estados financieros }\end{array}$} \\
\hline
\end{tabular}

Fuente: Elaboración propia siguiendo a Pulic (2004), Kujansivu y Lonnqvist (2007) y Laing et al. (2010).

\section{Metodología}

El presente trabajo de investigación posee las siguientes características:

- El análisis de los datos cuantitativos es paramétrico ya que se llevan a cabo pruebas tales como el coeficiente de correlación de Pearson y la regresión lineal que permiten describir la relación entre las variables, estimando el efecto de una variable sobre otra. Estos análisis se desarrollan tomando como referencia los estudios empíricos desarrollados por Mehri, Umar, Saeidi, Hekmat y Naslmosavi (2013); Mondal y Ghosh (2012); Wah, Hang y Wu (2011); Villalonga (2004) y Black, Jang y Kim (2006).

- El estudio es correlacional-causal ya que se han formulado 6 hipótesis para demostrar la relación que existe entre el VAIC ${ }^{\mathrm{TM}}$ y sus componentes, CEE, HCE y SCE, como variables formativas, con el rendimiento sobre activo total (ROA), como medida financiera, el Market to Book (MTB) como valor de capitalización de mercado de la empresa y la Q de Tobin (Q) como el nivel del valor de la acción (sobrevaluada/subvaluada), teniendo como variables de control el tamaño 


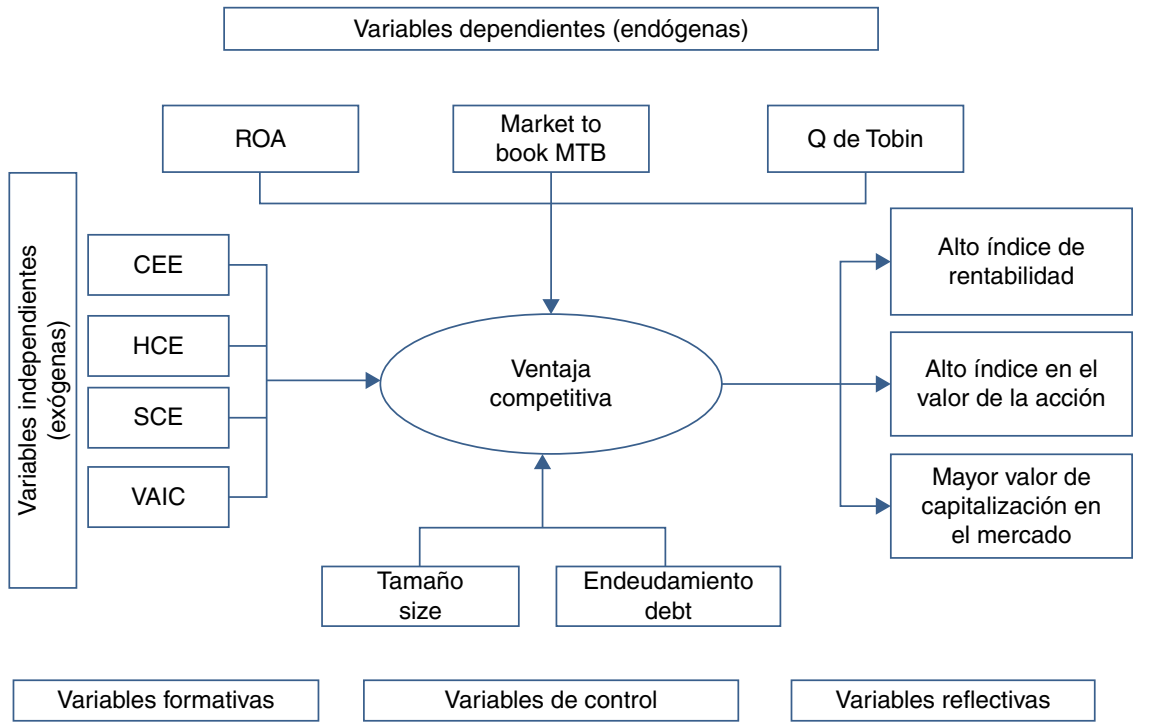

Figura 1. Modelo conceptual.

Fuente: Elaboración propia.

de las empresas mexicanas (SIZE) y el nivel de endeudamiento (DEBT) que conforman la muestra, con un nivel explicativo, proponiendo un sentido de entendimiento de las relaciones entre tales variables (véase figura 1).

- Para la validación de los modelos se examinaron los supuestos a través del estadístico DurbinWatson con lo cual se comprueba que los residuos son independientes entre sí, el diagnóstico de colinealidad aplicando los estadísticos de tolerancia y los factores de inflación de la varianza (FIV), el test de heterocedasticidad a través de la estimación consistente de White para asegurar que la varianza de los residuos es constante para cada valor de la combinación de las variables independientes.

- La base de datos se construyó a través de los informes financieros publicados por las 32 empresas mexicanas que cotizan en la Bolsa Mexicana de Valores (Bolsa Mexicana de Valores, 2014), los cuales brindan confiabilidad, validez y objetividad al ser cifras auditadas por firmas reconocidas a nivel nacional e internacional, y sirven para probar las hipótesis con base en una medición numérica y análisis estadístico empleando el programa SPSS versión 20.

\section{Modelo conceptual}

Al momento de diseñar el modelo conceptual para este trabajo de investigación se han establecido 4 variables independientes, 3 variables dependientes y 2 variables de control como se observa en la figura 1.

\section{Hipótesis}

A partir de la realización de diferentes estudios empíricos (Mehri et al., 2013; Mondal y Ghosh, 2012 y Wah et al., 2011) se ha comprobado cómo el capital intelectual guarda una relación positiva con el desempeño corporativo, por lo que es considerado como un factor determinante para generar 
Tabla 5

Listado de variables independientes

\begin{tabular}{lll}
\hline Variable & & Descripción conceptual (DC) y operacional (DO) de la variable \\
\hline CEE & DC & $\begin{array}{l}\text { La eficiencia del capital empleado: indicador de la eficiencia del valor añadido (VA) } \\
\text { del capital empleado (Pulic, 2000) }\end{array}$ \\
HCE & DO & $\begin{array}{l}\text { VA/CE } \\
\text { La eficiencia del capital humano: indicador de la eficiencia del valor añadido (VA) del capital } \\
\text { humano (Pulic, 2000) }\end{array}$ \\
& DO & VA/HC \\
SCE & DC & $\begin{array}{l}\text { La eficiencia del capital estructural: indicador de la eficiencia del valor añadido VA del capital } \\
\text { estructural. (Pulic, 2000) }\end{array}$ \\
& DC/VA \\
VAIC & DC & $\begin{array}{l}\text { Coeficiente de valor añadido intelectual. Metodología diseñada para proporcionar } \\
\text { información sobre el valor de eficiencia de la empresa y de su capital intelectual en la creación } \\
\text { de valor añadido de los activos tangibles e intangibles (Pulic, 2000) } \\
\text { CEE + HCE + SCE }\end{array}$ \\
& DO &
\end{tabular}

Fuente: Elaboración propia siguiendo a Pulic, 2000.

ventaja competitiva. El VAIC ${ }^{\mathrm{TM}}$ y sus componentes (CEE, HCE y SCE) han sido propuestos como indicadores que pretenden medir la habilidad intelectual de las empresas, a partir de los cuales se plantean las siguientes 6 hipótesis de investigación:

H1. Entre mayor sea el nivel del VAIC ${ }^{\mathrm{TM}}$ en las empresas del sector industrial la rentabilidad financiera (ROA) será más alta.

H2. Entre mayor sea el nivel del VAIC ${ }^{\mathrm{TM}}$ en las empresas del sector industrial su capitalización de mercado (MTB) será más alta.

H3. Entre mayor sea el nivel del $\mathrm{VAIC}^{\mathrm{TM}}$ en las empresas del sector industrial, el nivel del valor de la acción $(\mathrm{Q})$ será más alto.

H4. Entre mayor sean los nivel de los índices de CEE, HCE y SCE en las empresas del sector industrial la rentabilidad financiera (ROA) será más alta.

H5. Entre mayor sean los nivel de los índices de CEE, HCE, y SCE en las empresas del sector industrial su capitalización de mercado (MTB) será más alta.

H6. Entre mayor sean los nivel de los índices de CEE, HCE y SCE en las empresas del sector industrial, el nivel del valor de la acción $(\mathrm{Q})$ será más alto.

\section{Variables: independientes y dependientes}

En la tabla 5 se presentan como variables independientes al VAIC ${ }^{\mathrm{TM}}$ y sus componentes (CEE, HCE y SCE) calculadas a partir de datos publicados y auditados por las empresas que conforman el estudio.

En la tabla 6 se detallan las variables dependientes las cuales reflejan los resultados financieros de cada empresa, estas son: la ROA, el MTB y la Q de Tobin.

En la tabla 7 se explican las variables de control, que son SIZE y DEBT.

\section{Selección de la muestra: sector industrial en México}

En México el sector industrial representa un eje rector de crecimiento y desarrollo ya que emplea materias primas, energía, capital y mano de obra, generando empleos, fortaleciendo las 
Tabla 6

Listado de las variables dependientes

\begin{tabular}{|c|c|c|}
\hline \multicolumn{2}{|l|}{ Variable } & \multirow{2}{*}{$\begin{array}{l}\text { Descripción conceptual (DC) y operacional (DO) de la variable } \\
\text { Rendimiento sobre activo total (ROA) - return on asets -, mide la rentabilidad sobre los } \\
\text { activos de una empresa }\end{array}$} \\
\hline ROA & $\mathrm{DC}$ & \\
\hline & $\mathrm{DO}$ & Utilidad neta/Activo total \\
\hline MTB & DC & $\begin{array}{l}\text { Método de capitalización de mercado. Calcula el valor de una empresa mediante la } \\
\text { comparación de su valor contable a su valor de mercado }\end{array}$ \\
\hline & $\mathrm{DO}$ & Valor de mercado de las acciones/Capital contable -fondos propios- \\
\hline Q de Tobin $(\mathrm{Q})$ & DC & $\begin{array}{l}\text { Indicador bursátil que relaciona el precio de mercado de la acción con su valor patrimonial. Se } \\
\text { ha empleado para medir el desempeño eficaz o no del capital intelectual. Indica si la acción } \\
\text { está subvaluada }(\mathrm{Q} \text { de Tobin }<1) \text { o sobrevaluada }(\mathrm{Q} \text { de Tobin }>1) \text { con respecto a su valor } \\
\text { en libros. Mide la capacidad de la empresa para generar renta } \\
\text { (Pasivo total + valor mercado de las acciones)/Total activo }\end{array}$ \\
\hline
\end{tabular}

Fuente: Elaboración propia siguiendo a Ross, Westerfield y Jordan (2014); Molodchik y Bykova (2011) y Wah et al. (2011).

Tabla 7

Listado de las variables de control

\begin{tabular}{|c|c|c|}
\hline \multicolumn{2}{|l|}{ Variable } & \multirow{2}{*}{$\begin{array}{l}\text { Descripción conceptual (DC) y operacional (DO) de la variable } \\
\text { Valor de las ventas totales (Secretaría de Economía, 2009) }\end{array}$} \\
\hline Tamaño (SIZE) & $\mathrm{DC}$ & \\
\hline & DO & Ventas totales $/ 1,000,000,000$ \\
\hline Razón de endeudamiento (DEBT) & $\mathrm{DC}$ & $\begin{array}{l}\text { Razón de endeudamiento (deuda) que se utiliza como una medida } \\
\text { de porcentaje de cómo las empresas se llegan a financiar de sus } \\
\text { proveedores o acreedores. Mientras más baja sea esta medida, } \\
\text { mayor es la protección de los acreedores contra posibles pérdidas } \\
\text { en caso de quiebra o liquidación. Por otro lado, los empresarios se } \\
\text { benefician del apalancamiento que les ofrecen sus proveedores o } \\
\text { acreedores, sin poner en riesgo su propio capital }\end{array}$ \\
\hline & DO & Pasivo total/Activo total \\
\hline
\end{tabular}

Fuente: Elaboración propia siguiendo a Wah et al. (2011) y Ross et al. (2014).

cadenas productivas, incrementando la productividad e impulsando el sector servicios, configurando de esta manera nuevos escenarios de desarrollo económico. De acuerdo a cifras reportadas por el Banco Mundial (2014) el sector industrial mexicano aportó el 36\% del producto interno bruto en el año 2012.

En la tabla 8 se señalan las 32 empresas que conforman la muestra del sector industrial en base al subsector al que pertenecen y cuya población se ilustra en la figura 2. Cabe señalar que solamente se seleccionaron las empresas que han publicado el rubro de sueldos y salarios en sus estados financieros, por ser este un dato básico en el cálculo del VAIC ${ }^{\mathrm{TM}}$.

Se calcularon los promedios de las cifras correspondientes al periodo comprendido de 2006 a 2012 tomando como fuente primaria los montos auditados en los estados de pérdidas y ganancias, los balances generales y las notas a los estados financieros publicados por las empresas como información dirigida a sus accionistas.

\section{Resultados empíricos}

Para el presente trabajo de investigación se han construido 6 modelos de regresión lineal con su correspondiente descripción estadística y análisis de correlación, validando los supuestos a través 
Tabla 8

Caracterización de las empresas mexicanas del sector industrial

Sector industrial

\begin{tabular}{|c|c|c|}
\hline & Denominación social de la empresa: & Clave: \\
\hline \multicolumn{3}{|l|}{ Subsector: } \\
\hline \multirow[t]{3}{*}{ Bienes de equipo } & Alfa, SAB de CV & ALFA \\
\hline & Grupo Kuo, SAB de CV & KUO \\
\hline & Grupo Industrial Saltillo, SAB de CV & GISSA \\
\hline \multirow[t]{2}{*}{ Productos químicos } & Mexichem, SAB de CV & MEXCHEM \\
\hline & Grupo Pochteca, SAB de CV & POCHTEC \\
\hline Autopartes & San Luis Corporación, SAB de CV & SAN LUIS \\
\hline \multirow[t]{8}{*}{ Alimentos } & Grupo Bimbo, SAB de CV & BIMBO \\
\hline & Grupo Herdez, SAB de CV & HERDEZ \\
\hline & Grupo Industrial Maseca, SAB de CV & MASECAB \\
\hline & Grupo Minsa, SAB de CV & MINSA \\
\hline & Gruma, SAB de CV & GRUMA \\
\hline & Grupo Bafar, SAB de CV & BAFAR \\
\hline & Industrias Bachoco, $\mathrm{SAB}$ de $\mathrm{CV}$ & BACHOCO \\
\hline & Grupo Nutrisa, SAB de CV & NUTRISA \\
\hline \multirow[t]{6}{*}{ Metales, minería y aceros } & Industrias $\mathrm{CH}, \mathrm{SAB}$ de $\mathrm{CV}$ & $\mathrm{ICH}$ \\
\hline & G Collado, SAB de CV & COLLADO \\
\hline & Compañía Minera Autlán, SAB de CV & AUTLÁN \\
\hline & Grupo México, SAB de CV & GMEXICO \\
\hline & Industrias Peñoles, SAB de CV & PEÑOLES \\
\hline & Grupo Simec, SAB de CV & SIMEC \\
\hline \multirow[t]{3}{*}{ Materiales de construcción } & Corporación Moctezuma, SAB de CV & GMOCTEZ \\
\hline & Grupo Cementos de Chihuahua, SAB de CV & GCC \\
\hline & Internacional de Cerámica, SAB de CV & CERAMIC \\
\hline \multirow[t]{2}{*}{ Construcción } & Empresas ICA, SAB de CV & ICA \\
\hline & Grupo Mexicano de Desarrollo, SAB & GMD \\
\hline Confección de ropa & Edoardos Martin, SAB de CV & EDOARDO \\
\hline \multirow[t]{2}{*}{ Refresquero } & Arca Continental, SAB de CV & ARCA \\
\hline & Coca-Cola Femsa, SAB de CV & $\mathrm{KOF}$ \\
\hline Cervecería & Grupo Modelo, SAB de CV & GMODELO \\
\hline \multirow[t]{3}{*}{ Desarrollo inmobiliario } & Dine $\mathrm{SAB}$ de $\mathrm{CV}$ & DINE \\
\hline & Consorcio Hogar, SAB de CV & HOGAR \\
\hline & Desarrolladora Homex, SAB de CV & HOMEX \\
\hline
\end{tabular}

Fuente: Elaboración propia.

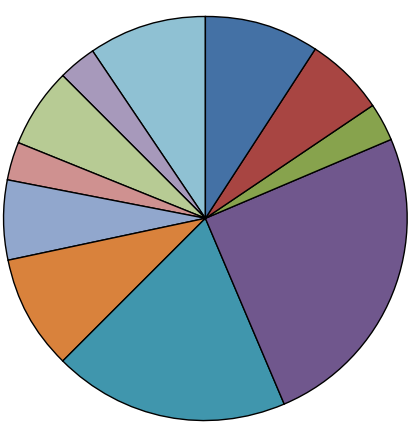

$\square$ Bienes de equipo (3)

$\square$ Productos químicos (2)

$\square$ Autopartes (1)

$\square$ Alimentos (8)

$\square$ Metales, minería y aceros (6)

$\square$ Materiales de construcción (3)

$\square$ Construcción (2)

$\square$ Confección de ropa (1)

$\square$ Refresquero (2)

$\square$ Cervecería (1)

$\square$ Desarrollo inmobiliario (3)

Figura 2. Muestra seleccionada de empresas mexicanas cotizadas a estudiar del sector industrial. Fuente: Elaboración propia. 
Tabla 9

Modelos de regresión lineal

\begin{tabular}{ll}
\hline Modelo & Ecuación de la regresión \\
\hline 1 & $\mathrm{ROA}_{\mathrm{i}}=\beta+\beta_{1} \mathrm{VAIC}^{\mathrm{TM}}+\beta_{2} \mathrm{SIZE}+\beta_{3} \mathrm{DEBT}+\varepsilon_{\mathrm{i}}$ \\
2 & $\mathrm{MTB}_{\mathrm{i}}=\beta+\beta_{1} \mathrm{VAIC}^{\mathrm{TM}}+\beta_{2} \mathrm{SIZE}+\beta_{3} \mathrm{DEBT}+\varepsilon_{\mathrm{i}}$ \\
3 & $\mathrm{Q}_{\mathrm{i}}=\beta+\beta_{1} \mathrm{VAIC} \mathrm{VIM}_{2} \mathrm{SIZE}+\beta_{3} \mathrm{DEBT}+\varepsilon_{\mathrm{i}}$ \\
4 & $\mathrm{ROA}=\beta+\beta_{1} \mathrm{CEE}+\beta_{2} \mathrm{HCE}+\beta_{3} \mathrm{SCE}+\beta_{4} \mathrm{SIZE}+\beta_{5} \mathrm{DEBT}+\varepsilon_{\mathrm{i}}$ \\
5 & $\mathrm{MTB}_{\mathrm{i}}=\beta+\beta_{1} \mathrm{CEE}+\beta_{2} \mathrm{HCE}+\beta_{3} \mathrm{SCE}+\beta_{4} \mathrm{SIZE}+\beta_{5} \mathrm{DEBT}+\varepsilon_{\mathrm{i}}$ \\
6 & $\mathrm{Q}_{\mathrm{i}}=\beta+\beta_{1} \mathrm{CEE}+\beta_{2} \mathrm{HCE}+\beta_{3} \mathrm{SCE}+\beta_{4} \mathrm{SIZE}+\beta_{5} \mathrm{DEBT}+\varepsilon_{\mathrm{i}}$ \\
\hline
\end{tabular}

Fuente: Elaboración propia.

Tabla 10

Descripción estadística de las empresas mexicanas del sector industrial

\begin{tabular}{lcccrrrrrr}
\hline & VAIC & CEE & \multicolumn{1}{c}{ HCE } & \multicolumn{1}{l}{ SCE } & \multicolumn{1}{c}{ ROA } & MTB & Q & SIZE & DEBT \\
\hline Mínimo & -3.162 & 0.042 & 0.839 & -5.142 & -0.151 & -1.464 & 0.543 & 0.001 & 0.150 \\
Máximo & 16.542 & 1.348 & 15.341 & 0.933 & 0.169 & 4.026 & 2.999 & 0.331 & 0.794 \\
Media & 4.030 & 0.291 & 3.410 & 0.330 & 0.045 & 1.638 & 1.416 & 0.075 & 0.464 \\
Desv. típ. & 3.392 & 0.236 & 2.883 & 1.048 & 0.073 & 1.106 & 0.580 & 0.091 & 0.182 \\
N & 32 & 32 & 32 & 32 & 32 & 32 & 32 & 32 & 32
\end{tabular}

Fuente: resultados obtenidos a partir de datos financieros de las empresas en el periodo 2006-2012 y procesados con el programa estadístico SPSS versión 20.

de los estadísticos Durbin-Watson, White y FIV, aceptando y/o rechazando total o parcialmente las hipótesis inicialmente planteadas, todo lo cual se desarrolla a continuación.

\section{Modelos de regresión}

Se han diseñado 6 modelos de regresión lineal (véase tabla 9) que corresponden a las hipótesis de investigación inicialmente planteadas con la finalidad de examinar la asociación del VAIC ${ }^{\mathrm{TM}}$ y sus componentes con los resultados obtenidos en el desempeño financiero, capitalización de mercado y la capacidad para incrementar el valor de la acción de las empresas que conforman la muestra, lo que permitirá la comprobación de las hipótesis dando paso a la ecuación lineal con fines predictivos.

A través de los modelos 1 a 3 se analiza la relación que existe entre el índice del VAIC ${ }^{\mathrm{TM}}$ y los indicadores financieros que miden la rentabilidad, la capitalización de mercado y el valor de mercado de la acción. En los siguientes 3 modelos (4-6) se emplean separadamente los componentes del VAIC ${ }^{\mathrm{TM}}$ (HCE, SCE y CEE) y los indicadores financieros que miden la rentabilidad, la capitalización de mercado y el valor de mercado de la acción, teniendo en todos ellos como variable de control el SIZE y el DEBT.

\section{Descripción estadística}

En la tabla 10 se muestran las estadísticas descriptivas de las variables independientes, dependientes y de control de las 32 empresas mexicanas del sector industrial durante el periodo 2006-2012.

Entre los valores obtenidos se observan la media, los mínimos y máximos, la desviación típica de los saldos promedios de los diferentes coeficientes calculados para la determinación del 
VAIC $^{\mathrm{TM}}$, las variables dependientes y de control. De conformidad con las cifras que integran la base de datos de las 32 empresas sujetas a estudio, y partiendo del valor de la media del VAIC ${ }^{\mathrm{TM}}$ que es de 4.030, 10 empresas se ubican por arriba de la media $(31.25 \%)$ y las otras $22(68.75 \%)$ por debajo. Las 5 primeras compañías que reportan el mayor índice en el promedio de los años 2006-2012 son CMOCTEZ con 16.5415, HOMEX con 10.082, HERDEZ con 8.7924, PEÑOLES con 7.1339 y GMEXICO con 6.366. Las compañías que tienen los 5 menores índices son HOGAR con -3.1615 , SANLUIS con 0.7299 , ICA con 0.8468 , SIMEC con 0.8677 y GCC con 1.6376 . Cabe resaltar que el comportamiento del $\mathrm{VAIC}^{\mathrm{TM}}$ refleja la misma tendencia en cada uno de sus componentes, estos son, en el CEE, HCE y SCE.

Con relación a las variables dependientes, la ROA tiene una media de 0.045 y por arriba de esta se ubican 18 empresas $(56.25 \%$ ) y 14 por debajo $(43.75 \%)$; entre las 5 empresas que reportan la mayor rentabilidad financiera se tienen a CMOCTEZ con 0.1688, GMEXICO con 0.1575, PEÑOLES con 0.1554, NUTRISA con 0.1412 y HERDEZ con 0.1299. Las que tienen menor rentabilidad financiera son HOGAR con -0.1509 , EDOARDO con -0.1360 , COLLADO con -0.0248 , POCHTEC con -0.0242 y SANLUIS con -0.0162 .

En cuanto al MTB se tiene una media de 1.638 y por encima de esta se encuentran 15 empresas $(46.88 \%)$ y 17 por debajo (53.12\%); entre las 5 empresas que reportan mayor capitalización de mercado están PEÑOLES con 4.0257, NUTRISA con 3.8257, BIMBO con 2.7414, CMOCTEZ con 2.6171 y HERDEZ con 2.2842. Con los 5 menores valores se tienen a HOGAR con -1.4642 , EDOARDO con 0.2957, GISSA con 0.5042, GMD 0.7642 y SAN LUIS con 0.7814.

Y respecto a la Q de Tobin la media es de 1.416; por encima de esta se encuentran 13 empresas $(40.63 \%)$ y 19 por debajo (59.37\%); entre las 5 empresas cuyo valor de acción se encuentra sobrevaluado se tienen a NUTRISA con 2.9986, PEÑOLES con 2.7829, CMOCTEZ con 2.3729, GMODELO con 2.2443 y POCHTEC con 2.0886; y las que no destacan por su capacidad para generar renta y cuya acción está subvaluada son: EDOARDO con 0.5429, GISSA con 0.7471, SIMEC con 0.8657 , GMD con 0.8971 y SANLUIS con 0.9257 .

Continuando con este análisis, al realizar un comparativo de las cifras iniciales de 2006 con las finales de 2012, las 5 empresas que mayor índice del VAIC ${ }^{\mathrm{TM}}$ reportan en 2006 son CMOCTEZ con 21.5256, HOMEX con 11.6352, HERDEZ con 9.5268, GMEXICO con 8.7868 y DINE con 7.3673; al compararlas con 2012, dichos índices reflejan una tendencia a la baja, es decir, CMOCTEZ 15.0507, HOMEX 9.1482, HERDEZ 8.4337, GMEXICO 5.8525 y DINE 1.5308. Lo mismo sucede con las empresas que tienen los menores índices en 2006 como son las empresas SANLUIS con 0.3948 , ICA con 0.7865, SIMEC con 1.7615, AUTLAN con 2.1684 y POCHTEC 2.2469; para el año 2012 la empresa EDOARDO reporta un índice negativo de -2.3536, SIMEC con 0.6008 , ICA con 0.8301 , ICH con 0.8313 , y GCC con 0.9296 . Conviene advertir que los valores del VAIC ${ }^{\mathrm{TM}}$ y los de sus componentes CEE, HCE y SCE reflejan una tendencia a la baja, es decir, han ido decreciendo en los últimos años en las empresas mexicanas del sector industrial en porcentajes que van de un 11 a un $30 \%$.

\section{Análisis de correlación}

Se llevó a cabo el análisis de la matriz de correlaciones con el cual se determinó el coeficiente de correlación entre las variables independientes VAIC ${ }^{\mathrm{TM}}$, CEE, HCE, SCE, y las dependientes ROA, MTB, Q, y de control SIZE y DEBT.

En la tabla 11, se presentan los resultados obtenidos al realizar el análisis de correlación de Pearson el cual revela que $3(8.33 \%)$ de las 36 correlaciones son significantes al nivel del $0.05 \mathrm{y}$ 
Tabla 11

Sector industrial mexicano: análisis de correlación de las variables independientes, dependientes y de control durante el periodo de 2006 a 2012

\begin{tabular}{|c|c|c|c|c|c|c|c|c|c|}
\hline & CEE & HCE & SCE & VAIC & ROA & МТВ & Q & SIZE & DEBT \\
\hline CEE & 1 & 0.094 & 0.257 & 0.229 & $0.452^{* * * *}$ & $0.588^{*}$ & $0.650^{*}$ & 0.200 & -0.235 \\
\hline $\mathrm{HCE}$ & & 1 & 0.295 & $0.948 *$ & $0.534^{*}$ & $0.385^{* *}$ & $0.487^{*}$ & 0.074 & $-0.319^{* * *}$ \\
\hline SCE & & & 1 & $0.578^{*}$ & $0.620^{*}$ & $0.628^{*}$ & 0.158 & 0.220 & $-0.328^{* * * *}$ \\
\hline VAIC & & & & 1 & $0.677^{*}$ & $0.562^{*}$ & $0.508^{*}$ & 0.145 & $-0.388^{* *}$ \\
\hline ROA & & & & & 1 & $0.679^{*}$ & $0.610^{*}$ & $0.386^{* *}$ & $-0.559^{*}$ \\
\hline MTB & & & & & & 1 & $0.807^{*}$ & $0.329^{* * * *}$ & -0.193 \\
\hline Q & & & & & & & 1 & 0.283 & -0.250 \\
\hline SIZE & & & & & & & & 1 & -0.073 \\
\hline DEBT & & & & & & & & & 1 \\
\hline
\end{tabular}

Fuente: resultados obtenidos a partir de datos financieros de las empresas en el periodo 2006-2012 y procesados con el programa estadístico SPSS versión 20.

* La correlación es significativa al nivel 0.01 (bilateral).

** La correlación es significante al nivel 0.05 (bilateral).

*** La correlación es significativa al nivel 0.1 (bilateral).

$16(44.44 \%)$ de las 36 correlaciones son significativas al nivel del 0.01 ; las variables que menos significación tienen son el SIZE y el DEBT.

A través de un examen visual se pueden evaluar los resultados presentados en la tabla 11, los cuales indican que la ROA mantiene una fuerte correlación con el VAIC ${ }^{\mathrm{TM}}\left(0.677^{* *}\right)$, un correlación media alta, positiva y significativa con el capital humano $(0.534 * *)$ y el capital estructural $\left(0.620^{* *}\right)$, y en menor medida con el capital empleado $\left(0.452^{* *}\right)$; el grado de correlación entre la ROA con el nivel de deuda es significativa pero negativa $(-0.559 * *)$, lo cual indica que al tener mayor DEBT una empresa, como consecuencia su rendimiento financiero se observará más bajo.

En relación con la variable MTB, que indica la capitalización de mercado de las empresas, se identifica una correlación moderadamente alta con el capital estructural $(0.628 * *)$, el capital empleado $\left(0.588^{* *}\right)$ y el índice de $\operatorname{VAIC}^{\mathrm{TM}}(0.562 * *)$, aunque con el capital humano el grado de correlación es débil $\left(0.385^{*}\right)$, pero significativo. El grado de correlación entre el MTB con el nivel de deuda es negativa y no significativa $(-0.193)$.

$\mathrm{Al}$ analizar la correlación de Pearson entre el nivel del valor de la acción Q y el VAIC ${ }^{\mathrm{TM}}$ y sus componentes, se observa una relación medianamente fuerte con el capital empleado $(0.650 * *)$, con el VAIC ${ }^{\mathrm{TM}}(0.508 * *)$ y el capital humano $(0.487 * *)$, no así con el capital estructural $(0.158)$.

La correlación que presentan los componentes CEE, SCE y HCE con el VAIC ${ }^{\mathrm{TM}}$ se destaca por ser positiva, moderadamente alta y significativa en los casos del capital estructural $\left(0.578^{* *}\right)$ y el capital humano $(0.948 * *)$, siendo este último el componente con mayor grado de correlación, lo cual es consistente con la teoría; cabe aclarar que no se muestra correlación significativa con el capital empleado (0.229) aun cuando forma parte de la integración del VAIC ${ }^{\mathrm{TM}}$.

En cuanto a la variable de control SIZE, esta no es una variable determinante en los niveles de CEE (0.200), HCE (0.074) y del VAIC ${ }^{\mathrm{TM}}(0.145)$, únicamente lo es en el desempeño financiero $(0.386 *)$.

Por último, el grado de correlación entre la variable DEBT con el VAIC ${ }^{\mathrm{TM}}\left(-0.388^{*}\right)$ es débil y con la ROA $\left(-0.559^{* *}\right)$ es moderadamente alta y negativa en ambas, lo cual es un indicador de que entre más endeudada este la empresa en alguna medida repercute negativamente en los niveles del VAIC ${ }^{\mathrm{TM}}$ y la ROA. 


\section{Resultados obtenidos a partir del análisis de los modelos de regresión lineal}

La tabla 12 contiene los resultados generados por cada uno de los análisis llevados a cabo en los 6 modelos de regresión lineal, de los cuales se puede destacar que todos son significativos, por lo cual son concluyentes en cuanto a afirmar que existe relación lineal entre las variables independientes y las variables dependientes en su conjunto, aunque se identifica que algunas son débiles como a continuación se explica.

El modelo 1, a través del índice del coeficiente intelectual de valor añadido, el SIZE de la empresa y el DEBT explica en su conjunto el 60.4\% de la varianza de la rentabilidad financiera, mientras que el modelo 2 solo lo hace en un $31.3 \%$ en relación con la capitalización de mercado; y el modelo 3 escasamente lo hace en un $23.2 \%$ sobre el valor de mercado de la acción.

En el modelo 4 las variables independientes y de control (los coeficientes de la CEE, la HCE, la SCE, el SIZE y el DEBT) explican escasamente en su conjunto el $16.0 \%$ de la varianza de la rentabilidad financiera, mientras que en el modelo 5 lo hacen en un $60.1 \%$ en relación con la capitalización de mercado y en el modelo 6 en un 58.2\% sobre el valor de mercado de la acción.

Estudios empíricos confirman estos resultados. De acuerdo a Mehri et al. (2013) el VAIC ${ }^{\text {TM }}$ explicó el $28.5 \%$ de la ROA y el 6.2\% del MTB, mientras que en el estudio realizado por Mondal y Ghosh (2012) el VAIC ${ }^{\mathrm{TM}}$ explicó del 6.3-35.4\% de la ROA en un periodo de tiempo comprendido de 1999 a 2008; en el estudio llevado a cabo por Wah et al. (2011) el VAIC ${ }^{\mathrm{TM}}$ explicó el $20 \%$ de la ROA y el 5.2\% del MTB. Al compararlo con los resultados obtenidos con las empresas mexicanas del sector industrial se puede observar que en México se genera un mayor porcentaje e influencia.

En la tabla 13 se presentan los resultados obtenidos de los coeficientes de regresión lineal de los 6 modelos por medio de los cuales se ha buscado explicar el impacto que tienen las

Tabla 12

Sector industrial mexicano: resultados de los modelos de regresión lineal durante el periodo de 2006 a 2012

\begin{tabular}{|c|c|c|c|c|c|}
\hline Modelo & $\mathrm{R}$ & $\mathrm{R}$ cuadrado & R cuadrado corregida & Error típ. de la estimación & $\mathrm{F}$ \\
\hline 1 & $0.802^{\mathrm{a}}$ & 0.642 & $0.604^{*}$ & 0.046 & 16.768 \\
\hline 2 & $0.616^{\mathrm{b}}$ & 0.380 & $0.313^{*}$ & 0.917 & 5.710 \\
\hline 3 & $0.553^{c}$ & 0.306 & $0.232^{* *}$ & 0.508 & 4.115 \\
\hline 4 & $0.544^{\mathrm{d}}$ & 0.296 & $0.160^{* * *}$ & 1.288 & 2.185 \\
\hline 5 & $0.816^{\mathrm{e}}$ & 0.665 & $0.601^{*}$ & 0.699 & 10.339 \\
\hline 6 & $0.806^{\mathrm{f}}$ & 0.649 & $0.582^{*}$ & 0.375 & 9.627 \\
\hline
\end{tabular}

Fuente: resultados obtenidos a partir de datos financieros de las empresas en el periodo 2006-2012 y procesados con el programa estadístico SPSS versión 20.

* La correlación es significativa al nivel 0.01 .

** La correlación es significante al nivel 0.05 .

*** La correlación es significativa al nivel 0.1 .

a Variables predictoras: (constante), VAIC ${ }^{\mathrm{TM}}$, SIZE (ventas), DEBT (endeudamiento). Variable dependiente: ROA.

b Variables predictoras: (constante), VAIC ${ }^{\mathrm{TM}}$, SIZE (ventas), DEBT (endeudamiento). Variable dependiente: MTB.

c Variables predictoras: (constante), VAIC ${ }^{\mathrm{TM}}$, SIZE (ventas), DEBT (endeudamiento). Variable dependiente: Q.

d Variables predictoras: (constante), CEE, HCE, SCE, SIZE (ventas), DEBT (endeudamiento). Variable dependiente: ROA.

e Variables predictoras: (constante), CEE, HCE, SCE, SIZE (ventas), DEBT (endeudamiento). Variable dependiente: MTB.

${ }^{\mathrm{f}}$ Variables predictoras: (constante), CEE, HCE, SCE, SIZE (ventas), DEBT (endeudamiento). Variable dependiente: Q. 
Tabla 13

Sector industrial en México: resultados de los coeficientes de regresión múltiple durante el periodo de 2006 a 2012

\begin{tabular}{|c|c|c|c|c|c|}
\hline \multirow[t]{2}{*}{ Modelo } & \multicolumn{2}{|c|}{ Coeficientes no estandarizados } & \multirow{2}{*}{$\begin{array}{l}\text { Coeficientes } \\
\text { tipificados } \\
\text { Beta }\end{array}$} & \multicolumn{2}{|r|}{$\mathrm{t}$} \\
\hline & $\mathrm{B}$ & Error típ. & & & \\
\hline \multirow[t]{4}{*}{1 (variable dependiente: ROA) } & (Constante) & 0.048 & 0.030 & & 1.571 \\
\hline & $\mathrm{VAIC}^{\mathrm{TM}}$ & 0.011 & 0.003 & $0.502^{*}$ & 4.059 \\
\hline & SIZE & 0.233 & 0.092 & $0.288^{*}$ & 2.525 \\
\hline & DEBT & -0.138 & 0.049 & $-0.343^{*}$ & -2.792 \\
\hline \multirow[t]{4}{*}{2 (variable dependiente: MTB) } & (Constante) & 0.599 & 0.607 & & 0.986 \\
\hline & $\mathrm{VAIC}^{\mathrm{TM}}$ & 0.176 & 0.053 & $0.539^{*}$ & 3.310 \\
\hline & SIZE & 3.090 & 1.837 & 0.253 & 1.683 \\
\hline & DEBTS & 0.212 & 0.983 & 0.035 & 0.216 \\
\hline \multirow[t]{4}{*}{3 (variable dependiente: $Q$ ) } & (Constante) & 1.085 & 0.337 & & 3.222 \\
\hline & $\mathrm{VAIC}^{\mathrm{TM}}$ & 0.078 & 0.029 & $0.455^{* *}$ & 2.642 \\
\hline & SIZE & 1.364 & 1.018 & 0.213 & 1.340 \\
\hline & DEBT & -0.182 & 0.545 & -0.057 & -0.335 \\
\hline \multirow[t]{6}{*}{4 (variable dependiente: ROA) } & (Constante) & -3.718 & 1.017 & & -3.654 \\
\hline & CEE & -0.250 & 0.378 & -0.121 & -0.663 \\
\hline & $\mathrm{HCE}$ & 0.392 & 0.414 & 0.186 & 0.948 \\
\hline & SCE & 0.575 & 0.487 & 0.210 & 1.179 \\
\hline & SIZE & -0.362 & 0.147 & -0.426 & -2.463 \\
\hline & DEBT & 0.323 & 0.582 & 0.100 & 0.556 \\
\hline \multirow[t]{6}{*}{5 (variable dependiente: MTB) } & (Constante) & -0.016 & 0.498 & & -0.032 \\
\hline & CEE & 2.142 & 0.565 & $0.457^{*}$ & 3.792 \\
\hline & $\mathrm{HCE}$ & 0.094 & 0.047 & $0.245^{* * *}$ & 2.000 \\
\hline & SCE & 0.486 & 0.134 & $0.460^{*}$ & 3.618 \\
\hline & SIZE & 1.576 & 1.437 & 0.129 & 1.097 \\
\hline & DEBT & 0.930 & 0.765 & 0.153 & 1.216 \\
\hline \multirow[t]{6}{*}{6 (variable dependiente: Q) } & (Constante) & 0.607 & 0.267 & & 2.270 \\
\hline & CEE & 1.520 & 0.303 & $0.619^{*}$ & 5.014 \\
\hline & $\mathrm{HCE}$ & 0.094 & 0.025 & $0.469^{*}$ & 3.736 \\
\hline & SCE & -0.097 & 0.072 & -0.175 & -1.348 \\
\hline & SIZE & 1.044 & 0.771 & 0.163 & 1.355 \\
\hline & DEBT & 0.000 & 0.410 & 0.000 & 0.000 \\
\hline
\end{tabular}

Fuente: resultados obtenidos a partir de datos financieros de las empresas en el periodo 2006-2012 y procesados con el programa estadístico SPSS versión 20.

* La correlación es significativa al nivel 0.01 (bilateral).

** La correlación es significante al nivel 0.05 (bilateral).

*** La correlación es significativa al nivel 0.1 (bilateral).

variables independientes y de control sobre las variables dependientes inicialmente planteadas en las hipótesis de investigación.

El modelo 1 explica la regresión lineal entre la ROA (variable dependiente) con el índice del VAIC ${ }^{\mathrm{TM}}$ (variable independiente), el SIZE y el DEBT (variables de control), concluyendo que el modelo presenta una relación moderadamente alta y significativa, observándose que el $\operatorname{VAIC}^{\mathrm{TM}}(\beta=0.502 *)$ y el SIZE $\left(\beta=0.288^{*}\right)$ son positivos, mientras que el DEBT se relaciona de forma negativa $\left(\beta=-0.343^{*}\right)$. Contrario a lo anterior, en el modelo 2 , solo el índice del $\operatorname{VAIC}^{\mathrm{TM}}\left(\beta=0.539^{*}\right)$ se relaciona de forma positiva y significativa con el nivel de capitalización de mercado de la empresa (MTB), las variables de control no impactan en este indicador. El 
Tabla 14

Sector industrial mexicano 2006-2012. Resumen de las pruebas de hipótesis basadas en los modelos de regresión

\begin{tabular}{|c|c|c|c|c|c|}
\hline Hipótesis de investigación & Conclusión & Modelo & $\begin{array}{l}\text { Explicación } \\
\text { en la variación } \\
\left(R^{2} \text { ajustada }\right)\end{array}$ & $\begin{array}{l}\text { Variable } \\
\text { de control }\end{array}$ & Observaciones \\
\hline $\begin{array}{l}\text { H1. A mayor nivel } \\
\text { del VAIC }{ }^{\mathrm{TM}} \text {, mayor } \\
\text { rentabilidad financiera } \\
\text { (ROA) }\end{array}$ & Sí & 1 & $0.604^{*}$ & $\begin{array}{l}\text { SIZE* } \\
\text { DEBT }^{*}\end{array}$ & $\begin{array}{l}\text { El VAIC }{ }^{\mathrm{TM}} \text { y el } \\
\text { tamaño son } \\
\text { predictores } \\
\text { positivos, mientras } \\
\text { que el DEBT es } \\
\text { negativo }\end{array}$ \\
\hline $\begin{array}{l}\text { H2. A mayor nivel } \\
\text { del VAIC }{ }^{\mathrm{TM}} \text {, mayor } \\
\text { capitalización de mercado } \\
\text { (MTB) }\end{array}$ & Sí & 2 & $0.313^{*}$ & & $\begin{array}{l}\text { El VAIC }{ }^{\mathrm{TM}} \text { es un } \\
\text { predictor positivo } \\
\text { del MTB }\end{array}$ \\
\hline $\begin{array}{l}\text { H3. A mayor nivel del } \\
\text { VAIC }^{\mathrm{TM}} \text {, el nivel del valor } \\
\text { de la acción será más alto } \\
\text { (Q) }\end{array}$ & Sí & 3 & $0.232^{* * *}$ & & $\begin{array}{l}\mathrm{El} \mathrm{VAIC} \mathrm{VM}^{\mathrm{TM}} \text { es un } \\
\text { predictor positivo } \\
\text { de la Q }\end{array}$ \\
\hline $\begin{array}{l}\text { H4. A mayor nivel de CEE, } \\
\text { HCE y SCE, mayor } \\
\text { rentabilidad financiera } \\
\text { (ROA) }\end{array}$ & No & 4 & $0.1604^{*}$ & SIZE $^{* * *}$ & $\begin{array}{l}\text { Solo el tamaño } \\
\text { (SIZE) es un } \\
\text { predictor negativo } \\
\text { de la ROA }\end{array}$ \\
\hline $\begin{array}{l}\text { H5. A mayor nivel de CEE, } \\
\text { HCE y SCE, mayor } \\
\text { capitalización de mercado } \\
\text { (MTB) }\end{array}$ & Sí & 5 & $0.601^{*}$ & & $\begin{array}{l}\text { CEE, HCE Y SCE, } \\
\text { son predictores } \\
\text { positivos del MTB }\end{array}$ \\
\hline $\begin{array}{l}\text { H6. A mayor nivel de HCE, } \\
\text { CEE y SCE, el nivel del } \\
\text { valor de la acción será más } \\
\text { alto }(\mathrm{Q}) \text {. }\end{array}$ & Sí & 6 & $0.582^{*}$ & & $\begin{array}{l}\text { CEE y HCE, son } \\
\text { predictores positivos } \\
\text { de la Q }\end{array}$ \\
\hline
\end{tabular}

Fuente: Elaboración propia.

* La correlación es significativa al nivel 0.01 (bilateral).

** La correlación es significante al nivel 0.05 (bilateral).

*** La correlación es significativa al nivel 0.1 (bilateral).

mismo comportamiento se observa en el modelo 3, donde la variable dependiente, nivel del valor de la acción $(\mathrm{Q})$, se relaciona de forma positiva y significativa únicamente con el VAIC ${ }^{\mathrm{TM}}$ $\left(\beta=0.455^{* *}\right)$.

En los modelos 4, 5 y 6, se toman los componentes del VAIC ${ }^{\mathrm{TM}}$ de forma separada para analizar la relación lineal y las variables de impacto. En el modelo 4 se observa que solo el SIZE $\left(\beta=-0.426^{* *}\right)$ incide de forma negativa en la ROA, es decir, entre mayor sea el SIZE de la empresa la ROA será menor. Al tomar el MTB como variable dependiente en el modelo 5, los resultados son concluyentes en cuanto a la relación positiva y significativa de los índices de CEE $\left(\beta=0.457^{*}\right), \operatorname{HCE}\left(\beta=0.245^{* * *}\right)$ y SCE $\left(\beta=0.460^{*}\right)$, mientras que el SIZE y el DEBT no tienen incidencia. Finalmente en el modelo 6 se destaca que las variables que contribuyen al nivel del valor de la acción son el capital empleado $\left(\beta=0.619^{*}\right)$ seguido del capital humano $\left(\beta=0.469^{*}\right)$, mientras que el SIZE y el DEBT no tienen incidencia.

Después de analizar los 6 modelos de regresión lineal, en la tabla 14 se presenta una recopilación de los resultados obtenidos, en donde las hipótesis H1, H2, H3, H5 y H6 se aceptan totalmente y la $\mathrm{H} 4$ se rechaza. 
Tabla 15

Estadístico de independencia Durbin-Watson

\begin{tabular}{llll}
\hline Modelo & \multicolumn{1}{l}{ F } & Sig. & Durbin-Watson \\
\hline 1 & 16.768 & 0.000 & 2.063 \\
2 & 5.710 & 0.004 & 2.004 \\
3 & 4.115 & 0.015 & 2.547 \\
4 & 2.185 & 0.087 & 1.478 \\
5 & 10.339 & 0.000 & 1.721 \\
6 & 9.627 & 0.000 & 1.963 \\
\hline
\end{tabular}

\section{Validación de los modelos}

En cuanto a la validez de los modelos analizados en términos de independencia (estadístico Durbin-Watson) y la no-colinealidad (estadísticos de nivel de tolerancia y los FIV y de homocedasticidad (estadístico F y test de White), a continuación se presentan los resultados que permiten validar los supuestos principales de los modelos y por ende a las hipótesis.

A partir del estadístico Durbin-Watson se valida el grado de independencia que existe entre los residuos ${ }^{1}$, asegurando que no exista autocorrelación positiva o negativa entre las variables. En la tabla 15 se observa que los valores en todos los modelos están entre 1.478 y 2.547 , por lo que se puede asegurar la existencia de independencia en las variables predictoras, validando dicho supuesto.

Con el fin de comprobar que no existe relación lineal exacta entre las variables independientes se utilizó el diagnóstico de colinealidad, aplicando los estadísticos de tolerancia y los FIV ${ }^{2}$. En la tabla 16 se puede observar que los valores de tolerancia son altos, lo cual indica que solo una porción muy baja de la variable dependiente está en función del resto de las variables independientes; aunado a lo anterior los valores del FIV son pequeños por lo que la varianza de la variable es menor; por lo anterior se concluye que no existe colinealidad.

Para asegurar que la varianza de los residuos es constante, es decir, que haya homocedasticidad, se encontró que en todos los modelos, a excepción del modelo 4 que plantea como variable dependiente la rentabilidad financiera (ROA) y como variables independientes la CEE, la HCE y la SCE, el SIZE y el DEBT, el valor de la probabilidad asociado al estadístico F es mayor a 0.05 , por lo que se acepta la hipótesis nula de cada modelo, concluyendo que no existen problemas de heterocedasticidad. Cabe aclarar que el modelo 4 se corrigió determinando el logaritmo natural de cada una de sus variables utilizando el sistema estadístico E-views para así lograr el cumplimiento del supuesto de homocedasticidad (véase tabla 17).

\footnotetext{
${ }^{1}$ El valor del estadístico Durbin-Watson (DW) debe ser cercano a 2 para asumir que nos existe autorrelación (1.5 a 2.5), aunque algunos autores como Valderrey (2010, p. 219) aceptan que valores como 1.3 se pueden considerar cercanos a 2 y evidencia de independencia.

${ }^{2}$ El valor de tolerancia y su inverso el FIV son medidas comunes para evaluar la colinealidad de parejas o de múltiples variables, manteniendo como umbral de tolerancia 0.10 y un FIV no mayor a 10 (Hair, Anderson, Tatham y Black, 1999, p. 185).
} 
Tabla 16

Diagnóstico de colinealidad, tolerancia y FIV

\begin{tabular}{|c|c|c|c|}
\hline \multirow[b]{2}{*}{ Modelo: } & & \multicolumn{2}{|c|}{ Estadísticos de colinealidad } \\
\hline & & Tolerancia & FIV \\
\hline 1 & (Constante) & & \\
\hline \multirow[t]{3}{*}{$\left(\mathrm{ROA}^{\mathrm{a}}\right)$} & $\mathrm{VAIC}^{\mathrm{TM}}$ & 0.835 & 1.197 \\
\hline & SIZE & 0.979 & 1.022 \\
\hline & DEBT & 0.849 & 1.178 \\
\hline 2 & (Constante) & & \\
\hline \multirow[t]{3}{*}{$\left(\mathrm{MTB}^{\mathrm{a}}\right)$} & $\mathrm{VAIC}^{\mathrm{TM}}$ & 0.835 & 1.197 \\
\hline & SIZE & 0.979 & 1.022 \\
\hline & DEBT & 0.849 & 1.178 \\
\hline 3 & (Constante) & & \\
\hline \multirow[t]{3}{*}{$\left(Q^{a}\right)$} & $\mathrm{VAIC}^{\mathrm{TM}}$ & 0.835 & 1.197 \\
\hline & SIZE & 0.979 & 1.022 \\
\hline & DEBT & 0.849 & 1.178 \\
\hline 4 & (Constante) & & \\
\hline \multirow[t]{5}{*}{$\left(\mathrm{ROA}^{\mathrm{a}}\right)$} & CEE & 0.818 & 1.223 \\
\hline & HCE & 0.702 & 1.424 \\
\hline & SCE & 0.854 & 1.171 \\
\hline & SIZE & 0.906 & 1.104 \\
\hline & DEBT & 0.840 & 1.191 \\
\hline 5 & (Constante) & & \\
\hline \multirow[t]{5}{*}{$\left(\mathrm{MTB}^{\mathrm{a}}\right)$} & $\mathrm{CEE}$ & 0.886 & 1.129 \\
\hline & $\mathrm{HCE}$ & 0.857 & 1.167 \\
\hline & SCE & 0.795 & 1.257 \\
\hline & SIZE & 0.929 & 1.077 \\
\hline & DEBT & 0.815 & 1.227 \\
\hline 6 & (Constante) & & \\
\hline \multirow[t]{5}{*}{$\left(Q^{a}\right)$} & CEE & 0.886 & 1.129 \\
\hline & HCE & 0.857 & 1.167 \\
\hline & SCE & 0.795 & 1.257 \\
\hline & SIZE & 0.929 & 1.077 \\
\hline & DEBT & 0.815 & 1.227 \\
\hline
\end{tabular}

${ }^{\text {a }}$ Variable dependiente.

Tabla 17

Estimación consistente de White. Tests de heterocedasticidad

\begin{tabular}{lll}
\hline Modelo & Estadístico-F & Probabilidad \\
\hline 1 & 0.6845 & 0.7150 \\
2 & 0.5207 & 0.8438 \\
3 & 0.4592 & 0.8862 \\
4 & 2.2105 & 0.0886 \\
5 & 0.2557 & 0.9960 \\
6 & 0.4049 & 0.9620 \\
\hline
\end{tabular}

\section{Conclusiones}

El capital intelectual radica en el capital humano, el capital estructural y el capital relacional de las empresas del sector industrial en México, los cuales tiene que ver con los conocimientos, competencias y capacidades que poseen las personas, con procesos y procedimientos propios 
de toda organización así como con las relaciones y alianzas que se mantienen con clientes y proveedores, lo cual propicia y genera riqueza en estas empresas.

El modelo del VAIC ${ }^{\mathrm{TM}}$ en investigaciones empíricas ha probado ser una herramienta útil en la generación de información relacionada con la eficiencia en la creación de valor añadido de los activos tanto tangibles como intangibles de las empresas, asumiendo que el capital intelectual de una empresa influye positivamente en su desempeño, rentabilidad, capitalización y valor de mercado en el precio de sus acciones, y por ende en su competitividad.

El VAIC ${ }^{\mathrm{TM}}$ a través de sus 3 indicadores determina la SCE, la HCE y la CEE; a través de esta investigación se ha comprobado que el capital humano es la principal fuente de capital intelectual y factor de creación de valor añadido que trasciende en las empresas del sector industrial en México.

Se ha comprobado que el VAIC ${ }^{\mathrm{TM}}$ y los coeficientes CEE, HCE y SCE sí inciden e impactan positivamente en elementos claves como son la rentabilidad financiera ROA como se demostró en la hipótesis 1, la capitalización de mercado MTB revelado en las hipótesis 2 y 5 , y en el nivel del valor de la acción Q manifestado en las hipótesis 3 y 6 de las empresas del sector industrial en México, así como el SIZE de estas en la hipótesis 1, con lo cual se validan y se reconocen estas 5 hipótesis que afirman tal incidencia e impacto (no así la hipótesis 4). Asimismo, se ha probado que mientras una empresa tenga un mayor índice de, los valores de su capital intelectual se verán reducidos y afectados negativamente, como lo refleja la hipótesis 1.

Los resultados vertidos en esta investigación les brindan a los accionistas y directivos de las compañías cotizadas del sector industrial en México un sistema de medición de su capital intelectual, contemplando con ello una visión de valor añadido, permitiéndoles identificar y comparar sus componentes claves, tratando al capital humano -los trabajadores- como su principal fuente generadora de estos valores, en el entendido de que una empresa que tiene altos índices de capital intelectual -medidos a través del modelo $\mathrm{VAIC}^{\mathrm{TM}}$ - posee un mayor coeficiente de valor añadido y por ende un alto índice de rentabilidad (ROA), un mayor valor de capitalización en el mercado (MTB) y un mayor nivel del valor de la acción (Q).

De acuerdo a los coeficientes obtenidos en la medición del capital intelectual en el presente trabajo de investigación, se han podido identificar a las empresas del sector industrial en México que mayores índices poseen, destacando Corporación Moctezuma, SAB de CV, CMOCTEZ, perteneciente al subsector materiales para la construcción, como la empresa con el más alto índice de $\mathrm{VAIC}^{\mathrm{TM}}$ y por consiguiente, la que mayor rentabilidad financiera posee (ROA), y cuya capitalización de mercado (MTB) y nivel del valor de su acción (Q) se ubican en los primeros lugares. Industrias Peñoles, SAB de CV, PEÑOLES, perteneciente al subsector metales, minería y aceros, es la que mayor capitalización de mercado posee (MTB) y que reporta altos índices de VAIC $^{\mathrm{TM}}$, rentabilidad financiera (ROA) y valor de su acción $(\mathrm{Q})$. Grupo Herdez, SAB de $\mathrm{CV}$, HERDEZ, perteneciente al subsector alimentos, se posiciona con altos índices de VAIC ${ }^{\mathrm{TM}}$, rentabilidad financiera (ROA) y capitalización de mercado (MTB). Grupo México, SAB de CV, GMEXICO, perteneciente al subsector metales, minería y aceros, posee altos índices de VAIC ${ }^{\mathrm{TM}}$ y rentabilidad financiera (ROA).

Contrario a estos resultados, la empresa Consorcio Hogar SA de CV, HOGAR, del subsector desarrollo inmobiliario, posee los más bajos índices de VAIC ${ }^{\mathrm{TM}}$, la menor rentabilidad financiera (ROA) y la menor capitalización de mercado (MTB), acompañada por las empresas San Luis Corporación, SAB de CV, SANLUIS, del subsector autopartes, con los más bajos índices de VAIC $^{\mathrm{TM}}$, rentabilidad financiera (ROA), capitalización de mercado (MTB) y valor de su acción (Q), y Edoardos Martin, SAB de CV, EDOARDO, del subsector confección de ropa, con los más 
bajos índices de rentabilidad financiera (ROA), capitalización de mercado (MTB) y valor de su acción (Q).

En los últimos años (de 2006 a 2012) los índices del valor del capital intelectual de las empresas del sector industrial en México han ido en declive, lo cual revela que la gestión del conocimiento no ha sido promovida en ellas; por lo tanto, se puede afirmar que no se ha reconocido al capital intelectual como un activo intangible clave y estratégico en su desarrollo, lo cual es un indicador en sí mismo de que a los empresarios de este sector su capital humano no les resulta prioritario y por lo mismo no le destinan los recursos necesarios que incrementen su potencial e impacten en la generación de valor añadido, siendo urgente que los accionistas y directivos rediseñen sus estrategias de crecimiento y desarrollo incentivando al capital humano, quien es pieza clave en la creación de valor y generación de ventaja competitiva.

La aplicación del modelo VAIC ${ }^{\mathrm{TM}}$ se puede ver entorpecida por algunos inconvenientes como el que se tuvo en este trabajo de investigación ya que no todas las empresas cotizadas del sector industrial en México publican en sus estados financieros el importe de los sueldos y salarios devengados, y al ser este un elemento básico en la aplicación de esta metodología se limitó su alcance, reduciéndose a solo un porcentaje del total de la muestra original.

\section{Referencias}

Abdulsalam, F., Al-Qaheri, H. y Al-Khayyat, R. (2011). The intellectual capital performance of Kuwaiti banks: An application of VAIC ${ }^{\mathrm{TM}}$. iBusiness, 3, 88-96. http://dx.doi.org/10.4236/ib.2011.31014

Arias, J., Cruz, H., Pedraza, M., Ordoñez, A. y Herrera, L. (2007). Los escenarios de la gestión del conocimiento y el capital intelectual en los procesos de investigación. Signo y Pensamiento, 26(50), 62-83.

Banco Mundial (2014). Indicadores de desarrollo mundial. México, América Latina y el Caribe [en línea; consultado 24 Jun 2014]. Disponible en: http://datos.bancomundial.org/pais/mexico

Becerra-Fernández, I. y Sabherwal, R. (2005). Knowledge management at NASA-Kennedy Space Center. Int J Knowledge and Learning, 1(1-2), 159-170.

Black, B. S., Jang, H. y Kim, W. (2006). Does corporate governance predict firms' market values? Evidence from Korea. Journal of Law, Economics, and Organization, 22(2), 366-413.

Bolsa Mexicana de Valores (BMV) (2014). Listado de empresas emisoras. Información digitalizada. Informes anuales. Reportes anuales [en línea; consultado 28 Feb 2014]. Disponible en: http://www.bmv.com.mx/

Bossi, A., Fuentes, Y. y Serrano, C. (2005). Reflexiones en torno a la aplicación del capital intelectual en el sector público. Revista española de financiación y contabilidad, XXXIV(124), 211-245. http://www.tandfonline.com/doi/abs/ 10.1080/02102412.2005.10779547

Brooking, A. (1996). El capital intelectual: el principal activo de las empresas del tercer milenio. Barcelona: Edtorial Paidós.

Bueno, E., Salmador, M. y Merino, C. (2008). Génesis, concepto y desarrollo del capital intelectual en la economía del conocimiento: Una reflexión sobre el Modelo Intellectus y sus aplicaciones. Estudios De Economía Aplicada, 26(2), 43-63. http://hdl.handle.net/10486/669095

Díaz, M., de Liz, Y. y Rivero, S. (2009). El factor humano como elemento dinamizador del proceso empresarial en la gestión de la información y conocimiento. ACIMED, 20(5), 42-55. http://scielo.sld.cu/scielo. php?script=sci_arttext\&pid=S1024-94352009001100004\&lng=es

Drucker, P. (1993). Post-capitalist society. Nueva York: Harper Collins Publishers.

Drucker, P. (1996). Drucker, su visión sobre: la administración, la organización basada en la información, la economía, la sociedad. Barcelona: Norma.

Drucker, P. (1 de noviembre 2001). The next society. The Economist, 16.

Fontrodona, J. y Sison, A. J. (2007). Hacia una teoría de la empresa basada en el bien común. Empresa Y Humanismo, 10(2), 65-92.

Freeman, E. (1984). Strategic management: A stakeholder approach. Marshfield, MA: Pitman.

Freeman, E., Wicks, A. y Parmar, B. (2004). Stakeholder theory and the corporate objective revisited. Organization Science., 15(3), 364-369. http://dx.doi.org/10.1287/orsc.1040.0066

Hair, J., Anderson, R., Tatham, R. y Black, W. (2010). Análisis multivariante. Madrid: Pearson Prentice Hall. 
Jensen, M. y Meckling, W. (1976). The theory of the firm: Managerial behaviour, agency costs, and ownership structure. Journal of Financial Economics, 3(4), 305-360. http://dx.doi.org/10.1016/0304-405X(76)90026-X

Kochan, T. y Rubinstein, S. (2000). Toward a stakeholder theory of the firm: The saturn partnership. Organization Science, 11(4), 367-386. http://dx.doi.org/10.1287/orsc.11.4.367.14601

Kujansivu, P. y Lonnqvist, A. (2007). Investigating the value and efficiency of intellectual capital. Journal of Intellectual Capital, 8(2), 272-287. http://dx.doi.org/10.1108/14691930710742844

Laing, G., Dunn, J. y Hughes-Lucas, S. (2010). Applying the VAIC ${ }^{\mathrm{TM}}$ model to Australian Hotels. Journal of Intellectual Capital, 11(3), 269-283. http://dx.doi.org/10.1108/14691931011064545

Lev, B. (1999). Seeing is believening-A better approach to estimating knowledge capital. CFO magazine April 2000. http://dx.doi.org/10.1016/0165-4101(95)00410-6

Lev, B. y Sougiannis, T. (1996). The capitalization, amortization and value-relevance of R\&D. Journal of Accounting and Economics, 21, 107-138.

Llano, C. (1997). Dilemas éticos de la empresa contemporánea. México: Fondo de Cultura Económica.

Mehri, M., Umar, M. S., Saeidi, P., Hekmat, R. K. y Naslmosavi, S. (2013). Intellectual capital and firm performance of high intangible intensive industries: Malaysia evidence. Asian Social Science, 9(9), 146.

Molodchik, M. y Bykova, A. (2011). Applying the VAIC model to Russian industrial enterprises. Proceedings of The European Conference On Intellectual Capital, 268-277.

Monagas-Docasal, M. (2012). El capital intelectual y la gestión del conocimiento. Ingenieria Industrial, 33(2), 142-150. http://scielo.sld.cu/scielo.php?script=sci_arttext\&pid=S1815-59362012000200006\&lng=es\&nrm=iso

Mondal, A. y Ghosh, S. (2012). Intellectual capital and financial performance of Indian banks. Journal of Intellectual Capital., 13(4), 515-530. http://dx.doi.org/10.1108/14691931211276115

Palomo, M. (2003). La evaluación de los activos intangibles parte 1. Los modelos. Ingenierías, VI(20), 12-17.

Pulic, A. (1998). Measuring the performance of intellectual potential in knowledge economy. Paper presented at the 2nd World Congress on Measuring and Managing Intellectual Capital, McMaster University, Hamilton.

Pulic, A. (2000). VAIC ${ }^{\mathrm{TM}}$ - an accounting tool for IC management. International Journal of Technology management, 20(5), 702-714. http://dx.doi.org/10.1504/IJTM.2000.002891

Pulic, A. (2004). Intellectual capital - does it create or destroy value? Measuring Business Excellence, 8(1), 62-68. http://dx.doi.org/10.1108/13683040410524757

Ramírez, D. E. (2007). Capital intelectual: Algunas reflexiones sobre su importancia en las organizaciones. Pensamiento \& Gestión, (23), 131-152. http://www.redalyc.org/articulo.oa?id=64602306

Ross, A. (1973). The economic theory of agency: The Principal's problem. American Economic Review, 63(2), 134-139. http://www.jstor.org/stable/1817064

Ross, S., Westerfield, R. y Jordan, B. (2014). Fundamentos de finanzas corporativas. México: Editorial McGraw Hill.

Sánchez, A., Melián, A. y Hormiga, E. (2007). El concepto de capital intelectual y sus dimensiones. Investigaciones Europeas de Dirección y Economía de las Empresas, 13(2), 97-111. ISSN 1135-2523.

Stern, J., Stewart, G. y Chew, D. (1995). The EVA financial system. Journal of Applied Corporate Finance, 3(2), 38-55. http://dx.doi.org/10.1111/j.1745-6622.1995.tb00285.x

Sveiby, K-E. (2010). Methods for measuring intangible assets. Copyright (Karl-Erik Sveiby, enero 2001, actualizado 27 Abr 2010).

Valderrey, P. (2010). SPSS 17. Extracción del conocimiento a partir del análisis de datos. México: Alfaomega-Rama.

Vassolo, R., García, J. y Weisz, N. (2007). Motivación emprendedora y teoría de los stakeholders. Empresa Y Humanismo, 10(1), 209-231.

Villalonga. (2004). Intangible resources Tobin's Q, and sustainability of performance differences. Journal of Economic Behavior and Organization, 54, 205-230. http://dx.doi.org/10.1016/j.jebo.2003.07.001

Villarreal, R. (2003). La empresa competitiva sustentable en la era del capital intelectual: IFA (Inteligente en la organización, Flexible en la producción y Ágil en la comercialización). México: Editorial McGraw Hill.

Wah, S., Hang, K. y Wu, W. (2011). Charting intellectual capital performance of the gateway to China. Journal of Intellectual Capital, 12(2), 249-276. http://dx.doi.org/10.1108/14691931111123412 